\title{
Crowd Modeling and Simulation Technologies
}

\author{
SUIPING ZHOU, DAN CHEN, WENTONG CAI, LINBO LUO, MALCOLM YOKE \\ HEAN LOW and FENG TIAN \\ Nanyang Technological University \\ and
}

VICTOR SU-HAN TAY and DARREN WEE SZE ONG

Defense Science \& Technology Agency, Singapore

and

BENJAMIN D. HAMILTON

Technical Support Working Group, USA

\begin{abstract}
As a collective and highly dynamic social group, human crowd is a fascinating phenomenon which has been constantly concerned by experts from various areas. Recently, computer-based modeling and simulation technologies have emerged to support investigation of the dynamics of crowds, such as a crowd's behaviors under normal and emergent situations. This paper assesses the major existing technologies for crowd modeling and simulation. We first propose a two-dimensional categorization mechanism to classify existing work depending on the size of crowds and the timescale of the crowd phenomena of interest. Four evaluation criteria have also been introduced to evaluate existing crowd simulation systems from the point of view of both a modeler and an end-user.

We have discussed some influential existing work in crowd modeling and simulation regarding their major features, performance as well as the technologies used in these work. We have also discussed some open problems in the area. This paper will provide the researchers with useful information and insights on the state-of-the-art of the technologies in crowd modeling and simulation as well as future research directions.
\end{abstract}

Categories and Subject Descriptors: I.6.5 [Simulation and Modeling]: Model Developmentmodeling methodologies; I.6.8 [Simulation and Modeling]: Types of Simulation-animation; gaming; J.4 [Social and Behavioral Sciences]: —psychology; sociology

General Terms: Algorithm, Design, Experimentation, Human Factors

Additional Key Words and Phrases: Crowd simulation, crowd dynamics, human behavior, multiagent system

\section{INTRODUCTION}

Human crowd is a fascinating social phenomenon in nature. In some situations, a crowd of people shows well-organized structure and demonstrates tremendous constructive power. While in other situations, people in a crowd seem to abandon their social norms and become selfish animals. Numerous incidents/accidents with large crowd (see Figure 1) have been recorded in human history, and many have led to severe casualties and injuries [Crowd Dynamics Pte Ltd 2009a]. How to predict and control the behavior of a crowd upon various events is an intriguing question faced by many psychologists, sociologists, physicists and computer scientists. It is also a major concern of many government agencies.

A crowd is not simply a collection of individuals. The behavior of an individual may be affected by others in the crowd, which may depend on various physiolog- 
ical, psychological and social factors. A common observation on human crowd is that an individual may behave quite differently in a crowd as compared to acting individually. That is, an individual may be forced to behave in a manner that is deemed proper by the crowd in a given situation. Therefore, a crowd may exhibit highly complex dynamics. In general, pure mathematical approaches or analytic models are not adequate in characterizing the dynamics of a crowd.

In recent years, modeling and simulation technologies have been gaining tremendous momentum in investigating crowd dynamics. Various simulation architectures have been developed [Musse and Thalmann 2001; McKenzie et al. 2004; Pan et al. 2005; Bandini et al. 2007]; virtual environment representations have also been constructed for crowd simulations [Paris et al. 2006; Shao and Terzopoulos 2005b; Yersin et al. 2009]. To represent the behavior of a crowd, a number of behavior models have been proposed [Helbing et al. 2000; Hughes 2003; Batty et al. 2003; Sung et al. 2004; Pelechano et al. 2008; Arentze and Timmermans 2009] with different types of modeling approaches, such as flow-based models and agent-based models. To study or mimic the dynamics of a crowd, modelers have considered a number of physical factors, social factors, and psychological factors when characterizing crowds in their models. Crowd models may also concern different aspects of a crowd. Some work aims at the "external characteristics" of a crowd, such as appearance, poses or movement patterns, coordinated positions of individuals; and some other work focuses on how a crowd's social behaviors evolve over time upon some events.

Crowd modeling and simulation has now become a key design issue in many fields including military simulation, safety engineering, architectural design, and digital entertainment etc. Crowd simulations have been intensively used for realtime tactical military training, such as simulating the civilian behaviors and combat actions in peacekeeping scenarios. Constructing a pool of human individuals and their behaviors in a virtual environment has been applied to identify possible risks or failures in a broad range of emergency situations and facilitate the architecture design to ensure safety. Some commercial software, such as Massive [Massive Software 2009] and AI.implant [Presagis 2009], have been developed and successfully

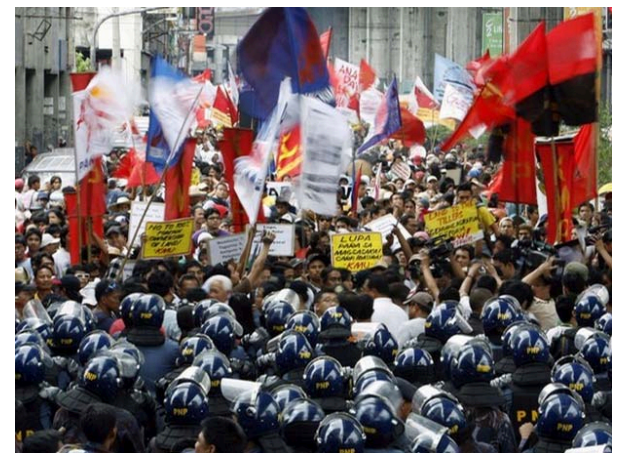

(A) A crowd in protest

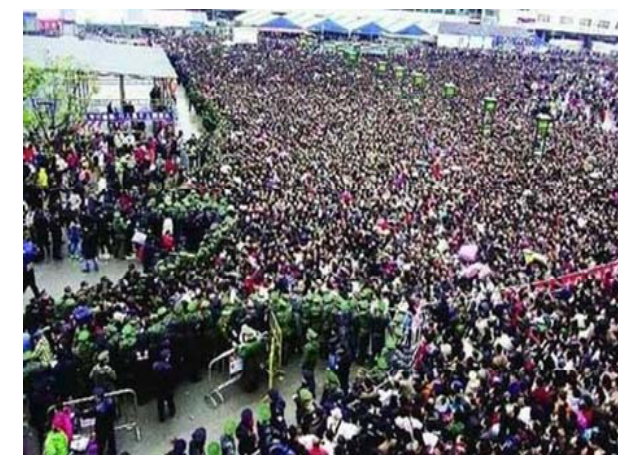

(B) A crowd stranded by the paralyzed transport system

Fig. 1. Examples of crowds. 
used in animating crowds for digital entertainment (e.g., scenes of crowd in Lord of the Rings, Age of Empires 3 etc).

Despite the many existing research efforts and applications in crowd modeling and simulation, it is still a young and emerging area. New researchers in this area are often bewildered by the many aspects that they need to consider when modeling the behavior of a crowd. Thus, there is a need to provide a comprehensive survey on crowd modeling and simulation technologies. There exists a few effort in summarizing crowd simulation models. For example, Santos and Aguirre gave a critical review of the existing crowd simulation models for emergency evacuation [Santos and Aguirre 2004]. However, their discussions are limited to those models for emergency evacuation situations, thus are not sufficient to cover the many behavioral aspects of human crowds in different situations. In addition, many new research and development efforts have been conducted in recent years. This paper serves as an up-to-date review of existing work in crowd modeling and simulation. We do not aim to give a complete account of all existing work. Instead, we will describe the major modeling features and related mechanisms of some representative work. We hope to provide readers with a clear overview of the state-of-the-art of the area as well as some concrete insights such as how different behavioral aspects could be modelled. To this end, it is necessary to have a clear classification of existing work. In fact, one of our major concerns while writing this paper is on the organization of existing work. Despite the variety in model types, behavioral factors involved and application areas, we find that the existing work in crowd modeling and simulation can be generally categorized by the size of the simulated crowd and/or the time-scale of the crowd phenomena of interest.

Simulated crowds in existing models may consist of thousands or even hundreds of thousands (huge-sized), hundreds (medium-sized), or tens (small-sized) of individuals. The crowd size often determines the types of approaches used to model a crowd. For example, existing work on modeling huge-sized crowd usually treats the crowd as a whole and focuses on the global trend of the crowd, due to the tremendous computational cost involved. For a small to medium-sized crowd, the relative small size of the crowd allows researchers to model the behavior of the individuals in the crowd. Such individual-based approaches usually build more details into the crowd model and support investigation of crowd dynamics at individual level.

As far as a crowd's time scale is concerned, the work in crowd modeling either concerns long-term or short-term crowd phenomena. Examples of long-term crowd simulations include the investigation on how people's opinions are affected by various factors, how extreme ideologies are formed and spread among the individuals in the crowd [Deffuant 2006; Salzarulo 2006]. The underlying process for these phenomena have a relatively long time period. In contrast, research in the short-term crowd simulation focuses on the short-term behavior of a crowd, which in general investigates how a crowd will respond to various events such as emergencies and threats, given the crowd composition and the social, psychological and physical characteristics of the individuals and groups in the crowd. The time-scale of the crowd phenomena of interest is usually in the order of minutes to hours.

Along the dimensions of crowd size and time scale, this paper gives an up-todate overview on crowd modeling and simulation technologies. The paper provides 
a review of models, features and applications of some major existing work in this area. As crowd modeling and simulation is still a new and rapidly evolving field, developments and research achievements from different communities tend to be diverse and address issues from different perspectives. Although it is difficult to fully assess and compare all the existing work in crowd modeling and simulation, the paper attempts to analyze and evaluate the major efforts in this area based on carefully defined evaluation criteria. The evaluation is intended to reveal potential improvements and open research problems in the future.

The remainder of the paper is organized as follows. Section 2 introduces the classifications and defines the evaluation criteria for analyzing crowd simulation systems. Section 3 explores major existing work in this field, which will be categorized based on the taxonomy and evaluated against the evaluation criteria. Section 4 discusses open research problems in crowd modeling and simulation. Section 5 summarizes observations on the work surveyed.

\section{CLASSIFICATIONS AND EVALUATION CRITERIA}

Existing crowd models and simulation systems can be roughly categorized using a two-dimensional taxonomy defined by crowd size and time scale. For the models and systems in each category, we further classify them based on two aspects. The first aspect is the modeling approach being used. These modeling approaches mainly include flow-based approach, particle system approach, and agent-based approach. The modeling approach may have significant impact on the simulated crowd behavior and also the runtime performance of the system. Thus it must be carefully chosen by a modeler. The other aspect is the behavioral factors being considered. These factors include tangible factors like physical and physiological characteristics of individuals (e.g., position, speed, etc.), and intangible factors like social factors and psychological factors (e.g., family tie, emotion, etc.).

To properly assess various crowd simulation systems, we introduce different types of evaluation criteria from two perspectives: the first type of criteria is from a modelers' point of view that concerns with the design and development of crowd models, the second type of criteria is from the simulation execution point of view that concerns runtime performance of simulation. Note that we do not include believability as an evaluation criterion in this paper. Although many researchers may argue that the ultimate evaluation of a crowd modeling and simulation work is to see whether the generated crowd behavior is believable or not, we feel that believability is not a properly defined concept - any claim on believability is subjective, thus is debatable and even doubtful.

\subsection{Model Classifications}

According to different crowd size and time-scale involved, we found that existing crowd models can be generally classified into three categories: models of longterm crowd phenomena, models of short-term phenomena of huge-sized crowd, and models of short-term phenomena of medium to small-sized crowd. Each category corresponds to a region in the 2D space defined by crowd size and time scale, as shown in Figure 2. The size and the time scale of a simulated crowd relate closely with the crowd model's modeling approaches and features. 
Different crowd modeling approaches may reproduce the feature, state and behavior of a real crowd in different granularity when modeling a crowd. Some approach treats the crowd as a whole and studies its dynamics at the crowd level. A typical example is the flow-based approach, which models the crowd as a continuous flow and usually aims at the movement pattern of the crowd [Hughes 2003]. A model for huge-sized crowd may have to opt for this type of approach due to the computational cost involved. Executing thousands of virtual individuals demands tremendous computing resources especially when each virtual individual is an intelligent and autonomous entity rather than a simple object. In contrast, a model aiming at medium to small-sized crowd often uses a modeling approach with much finer granularity. For example, each individual in the crowd may be modelled as an autonomous agent.

The time scale of a crowd phenomenon of interest is determined by the objective of a crowd simulation system which also confines its applicability. A crowd simulation model of a long-term phenomenon usually focus on some intangible social and psychological characteristics rather than the physical characteristics of the crowd. Research in this category is basically suitable for academic research in social science or high level operational studies, for instance, to study how extreme ideologies are formed and spread among individuals in the crowd. On the other hand, simulation models of short-term crowd phenomena usually describe crowds' physical characteristics, especially positions and movement patterns. Besides academic research, these models have been widely applied in military training, digital entertainment and daily operational studies.

2.1.1 Crowd Modeling Approaches. Crowd modeling approach reflects the overall simulation mechanism that controls the way how the simulated individual in the crowd performs. In the following, we discuss three major modeling approaches with different modeling granularities. The crowd may be treated as a whole, or as a collective of homogeneous/heterogeneous entities with interactions among them.

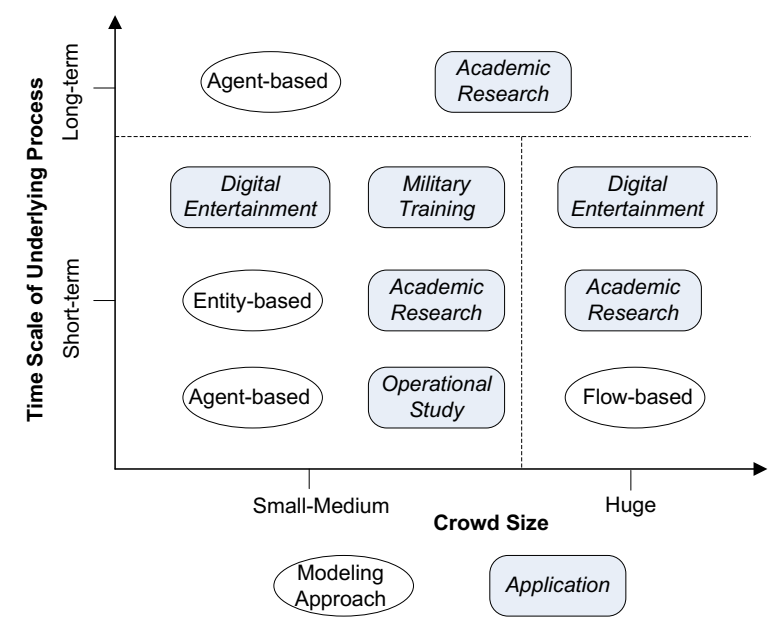

Fig. 2. Classification of crowd model. 
Flow-based Approach: The flow-based approach models a crowd as continuous flow of fluid (see Figure 3), such as EVACNET4 [Kisko et al. 1998] and Flow Tiles technique [Chenney 2004]. Due to the inherent nature of flow-based models, they basically neglected the features of individuals. In this sense, flow-based models are mainly useful in estimating the flow of movement/evacuation process for huge and dense crowds.

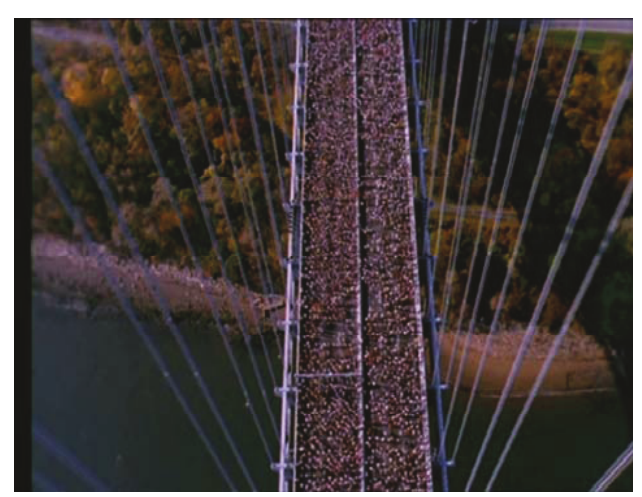

(A) A highly dense crowd in a marathon race

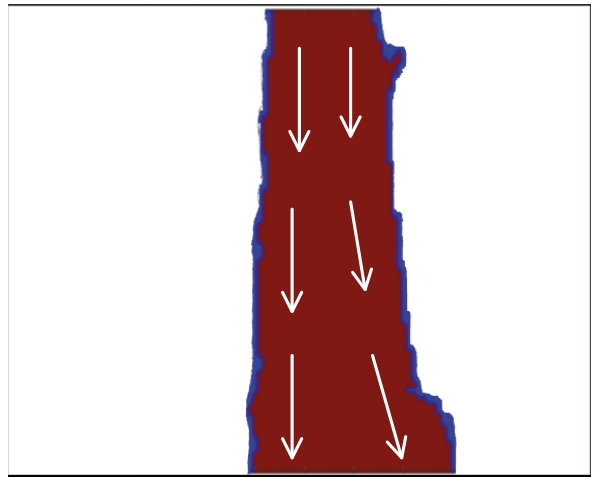

(B) Flow-based model of the crowd

Fig. 3. An example of flow-based crowd model. [Original pictures were retrieved from http://www.cs.ucf.edu/ sali/Projects/CrowdSegmentation/index.html.]

Entity-based Approach: Individuals are modeled as a set of homogeneous entities in this approach. A typical example of this approach is the particle system model that treats individuals in a crowd as particles in the physical world (see Figure 4), such as Helbing's social force model [Helbing et al. 2000]. In models that adopt the entity-based approach, the motions of the entities are usually influenced by some global/local laws that are introduced to represent various physical/social/psychological influences on an individual's movement in a crowd. Some global emerging phenomena such as jamming and flocking can be generated by these models.

Agent-based Approach: Agent-based approach models each individual in a crowd as an intelligent and autonomous agent, which may have capabilities to behave in the simulated world ranging from reacting to certain events to adapting to complex dynamic environment. Agents are regulated by sets of decision rules, and each agent may make decision independently. The decision rules for an agent are usually based on some local information that are relevant to the agent. Nevertheless, some sustainable global patterns may emerge even with some simple local rules. This approach also allows for more behavioral factors to be considered. The agent-based approach to crowd modeling and simulation has gained tremendous momentum recently due to the significant increase in computing power.

It should be noted that sometimes the difference between an entity-based model and an agent-based model is not very clear as researchers tend to incorporate more and more behavioral factors into entity-based models [Braun et al. 2005] and the 


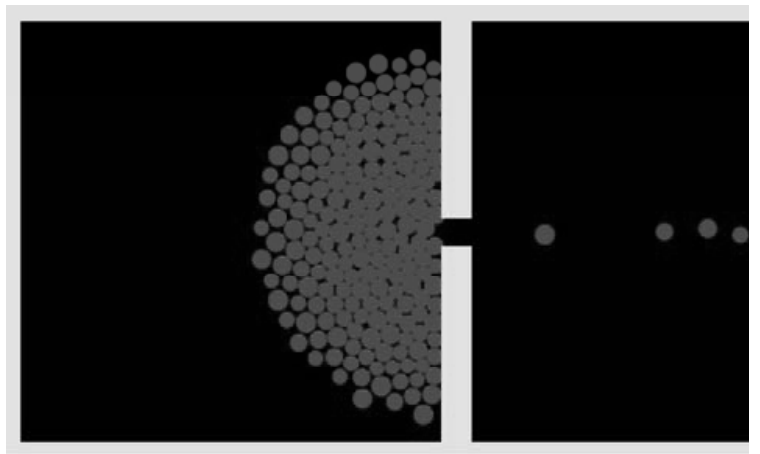

Fig. 4. An example of particle system model.[Original pictures were retrieved from reference [Helbing et al. 2000].]

agents in some agent-based crowd models are equipped with some rather simple rules. However, in general, an agent is more complex than an entity. An agent has its own attributes and states that represent various behavioral factors such as moving speed, emotion, social ties, etc. An agent also has certain level of cognitive and reasoning capability that allow it to sense its surroundings, assess the current situation and make decisions. While the homogeneous entities in an entity-based crowd model generally lack such capability - they simply react to the current situation in a similar manner according to some pre-defined global or local laws.

2.1.2 Behavioral Factors. The dynamics of a crowd is characterized and influenced by a number of factors, such as the position and moving speed of the individuals, various social relations among the individuals (e.g., family ties, leadership), and the emotional states of the individuals, etc. Based on different objectives and design requirements, different crowd models may take into account different behavioral factors. Here, we broadly divide these factors into three categories: (tangible) physical factors, (intangible) social factors, and (intangible) psychological factors.

Physical Factors: Physical factors refer to those external tangible characteristics of an individual such as position, moving speed, appearance, gesture, etc. A crowd model that considers only these tangible factors usually aims to investigate how the movement of an individual may be affected by the movement of others in the crowd. In general, physical factors are considered in most of the crowd models as the basic and low level inputs for decision making.

Social Factors: In addition to the tangible factors, human behavior is also influenced by a wide variety of social factors, such as culture, social norms, family ties and leadership etc. The computational models that incorporate these factors are usually based on social theories and observations from social studies. For example, Kaminka and Fridman adopted the social comparison theory in their crowd behavior model [Kaminka and Fridman 2006]. Pan et al [Pan et al. 2005] also employed a multi-agent based framework to demonstrate some emergent human social behaviors, for instance, competing, queuing, and herding.

Psychological Factors: As manifested by numerous studies, psychological factors such as emotion play an important role in human decision making. Due to 
the complex process involved, there is still no consensus on how human behavior is influenced by these factors. The crowd models that take into account these factors are in general fragmented and immature. However, research in this area is getting momentum recently. For example, Gratch and Marsella [Gratch and Marsella 2004] have proposed a framework for modeling emotion based on the appraisal theory.

\subsection{Evaluation Criteria}

In order to produce a realistic crowd model and execute the simulation efficiently and effectively, a crowd simulation system should meet certain requirements from computer engineering's perspective. In this paper, we abstract these requirements as several evaluation criteria, which are grouped into two major categories.

The first category is from a modeler's point of view that concerns the design and development of crowd models, which includes flexibility and extensibility.

Flexibility: Flexibility refers to a model's capability to adapt to different situations. A model is flexible if it is able to embrace potential internal or external changes in accordance to various situations, especially when the changes are uncertain. A major problem with many existing crowd models is that these models are too tightly coupled with some specific situations. For example, they may adopt some decision rules specific to a given situation that makes it hard to adapt to other situations.

Extensibility: A model is extensible if it is able to accommodate new features without much difficulty. For example, an extensible behavior model should be able to incorporate new behavioral factors into the existing decision making process without much difficulty.

The second category is from the simulation execution point of view that concerns the runtime performance of a crowd simulation model, which includes execution efficiency and scalability.

Execution Efficiency: Execution efficiency mainly concerns the time needed to execute a crowd simulation for a given scenario. For a realtime crowd simulation, this is also reflected by the delay that the simulation system responds to the user interaction. Obviously, the shorter the execution time is required (or the smaller the delay is incurred), the better execution efficiency a crowd simulation has. Therefore, a detailed model taking into account many behavioral factors may not be regarded as a good model if its execution efficiency is too low. A modeler needs to carefully balance a model's granularity and its computational cost. A trade-off is usually necessary for crowd with large size.

Scalability: Scalability is an important issue for crowd simulation especially for large-sized crowd. Many crowd models adopt some decision rules that need to determine the interaction among neighboring individuals. Thus, the computational overhead often increases very fast as the size of the crowd increases, which will make the simulation very slow. Scalability has been widely studied in the parallel computing and distributed simulation community, and various methods have been proposed to enhance a system's scalability. However, it should be pointed out that scalability is largely ignored in the existing crowd simulation systems. 


\section{EXISTING WORK}

As an intriguing issue, crowd behavior has been studied by researchers in different areas, e.g., social scientists, psychologists, physicists, computer scientists, etc. Since the last decade, modeling and simulation technology has become an important tool in the study of crowd behavior with quite a number of crowd behavior modeling approaches being proposed and a lot of crowd simulation systems being built. Our aim in this section is not to give a complete account of all existing work. Instead, we will sample some representative work in crowd modeling and simulation, as listed in Table I. These work are selected according to our model classification methodology as described in Section 2. First, we will discuss some existing work that focus on the long-term phenomenon of crowd. Secondly, we will discuss work on the investigation of short-term phenomenon of huge-sized crowd. Thirdly, we will discuss the modeling and simulation efforts on small-to-medium sized crowd with short-term phenomenon. We further classify work in this category according to the modeling approach being used, namely, entity-based approach and agentbased approach. As agent-based approach represents the major trend in crowd modeling and simulation, a significant part of our discussion is on work with agentbased approach. In particular, we put emphasis on work involving virtual crowds in urban environment, which perhaps represent the most demanding area for crowd modeling and simulation in terms of the level of detail (e.g, behavioral factors) of the crowd/individual model as well as the computational resources needed.

For the work in each category, we will summarize their major design considerations, and discuss how these considerations were addressed with various behavior modeling features and mechanisms. Depending on different application areas and different objectives of simulation study, the results of crowd simulations may be presented in different ways such as values of some key statistics, 2D plots, 2D/3D animation, etc. Nevertheless, animation has become increasingly important for many important applications, especially for applications using agent-based crowd models. In fact, an important validation method relies on users' perception on the animated crowd behavior. Therefore, for some work on agent-based crowd modeling and simulation, we will also briefly discuss how the simulation models facilitate crowd animation.

We will also make comments on the existing work using the proposed evaluation criteria. It should be noted that our aim is not to compare these existing work since different work may have different applications and objectives. Through our analysis, we hope to provide researchers and developers in crowd modeling and simulation with more concrete insights such as what types of models are more suitable for what type of applications involving human crowds, how different behavioral factors could be modelled, etc.

\subsection{Modeling and Simulation of Long-term Crowd Phenomenon}

The long-term crowd phenomenon has been studied mainly by social scientists. Social scientists have been intrigued by questions like how social groups are formed, how extreme ideologies are formed and spread among individuals in a population, etc. The crowd phenomena of interest in these questions often have a relatively long time period, e.g., several days to several years. A set of social theories, e.g., 
Table I. Influential Research Work on Crowd Modeling and Simulation

\begin{tabular}{|c|c|c|c|}
\hline $\begin{array}{l}\text { Organizations / Research } \\
\text { Groups }\end{array}$ & $\begin{array}{l}\text { Model Abbrevi- } \\
\text { ation }\end{array}$ & Nature of Work & $\begin{array}{l}\text { Application } \\
\text { Area }\end{array}$ \\
\hline $\begin{array}{lr}\text { Guillaume } & \text { Deffuant at } \\
\text { Laboratoire } & \text { d'Ingnierie } \\
\text { des Systmes } & \text { Complexes, } \\
\text { Cemagref } & \\
\end{array}$ & Deffuant & $\begin{array}{ll}\text { Long Term } & \text { Crowd } \\
\text { Process } & \end{array}$ & Social Study \\
\hline $\begin{array}{llr}\text { Laurent } & \text { Salzarulo at } \\
\text { Université } & \text { de Lausanne, } \\
\text { Institut de mathématiques } \\
\text { appliquées }\end{array}$ & Salzarulo & $\begin{array}{lll}\text { Long } & \text { Term } & \text { Crowd } \\
\text { Process } & & \end{array}$ & Social Study \\
\hline $\begin{array}{l}\text { Thomas M. Kisko et al at } \\
\text { University of Florida }\end{array}$ & EVACNET4 & $\begin{array}{l}\text { Movement of Large } \\
\text { Crowd in Complex } \\
\text { Urban Environment }\end{array}$ & Civil Planning \\
\hline $\begin{array}{l}\text { Computer Graphics Group } \\
\text { at University of Wisconsin, } \\
\text { Madison }\end{array}$ & Flow Tiles & $\begin{array}{l}\text { Movement of Large } \\
\text { Crowd in Complex } \\
\text { Urban Environment }\end{array}$ & $\begin{array}{l}\text { Digital Enter- } \\
\text { tainment }\end{array}$ \\
\hline $\begin{array}{l}\text { Adrien Treuille et al at Uni- } \\
\text { versity of Washington }\end{array}$ & $\begin{array}{l}\text { Continuum } \\
\text { Crowds }\end{array}$ & $\begin{array}{l}\text { Movement of Large } \\
\text { Crowd in Complex } \\
\text { Urban Environment }\end{array}$ & $\begin{array}{l}\text { Digital Enter- } \\
\text { tainment }\end{array}$ \\
\hline $\begin{array}{l}\text { Dirk Helbing at Dresden Uni- } \\
\text { versity of Technology }\end{array}$ & Helbing & $\begin{array}{lr}\text { Movement } & \text { of } \\
\text { Medium } & \text { Crowd } \\
\text { in Simple } & \text { Environ- } \\
\text { ment } & \\
\end{array}$ & $\begin{array}{l}\text { Operational } \\
\text { Study }\end{array}$ \\
\hline $\begin{array}{l}\text { Institute of Theoretical } \\
\text { Physics at University of } \\
\text { Cologne }\end{array}$ & ITP@UC & $\begin{array}{lr}\text { Movement } & \text { of } \\
\text { Medium } & \text { Crowd } \\
\text { in Simple } & \text { Environ- } \\
\text { ment } & \\
\end{array}$ & $\begin{array}{l}\text { Operational } \\
\text { Study }\end{array}$ \\
\hline $\begin{array}{l}\text { Center for Advanced Spatial } \\
\text { Analysis at University Col- } \\
\text { lege London }\end{array}$ & CASA@UCL & $\begin{array}{lr}\text { Movement } & \text { of } \\
\text { Medium } & \text { Crowd } \\
\text { in Complex } & \text { Envi- } \\
\text { ronment } & \\
\end{array}$ & Civil Planning \\
\hline Crowd Dynamics Pte Ltd & $\begin{array}{l}\text { Legion@Crowd } \\
\text { Dynamics }\end{array}$ & $\begin{array}{lr}\text { Movement } & \text { of } \\
\text { Medium } & \text { Crowd } \\
\text { in Urban } & \text { Environ- } \\
\text { ment } & \\
\end{array}$ & Civil Planning \\
\hline $\begin{array}{l}\text { Virtual Reality Lab at the } \\
\text { Swiss Federal Institute of } \\
\text { Technology }\end{array}$ & VRLab@EPFL & $\begin{array}{lr}\text { Movement } & \text { of } \\
\text { Medium } & \text { Crowd } \\
\text { in Urban } & \text { Environ- } \\
\text { ment } & \end{array}$ & $\begin{array}{l}\text { Digital Enter- } \\
\text { tainment }\end{array}$ \\
\hline $\begin{array}{l}\text { Media Research Lab at New } \\
\text { York University }\end{array}$ & MRL@NYU & $\begin{array}{lr}\text { Movement } & \text { of } \\
\text { Medium } & \text { Crowd } \\
\text { in Urban } & \text { Environ- } \\
\text { ment } & \end{array}$ & $\begin{array}{l}\text { Digital Enter- } \\
\text { tainment }\end{array}$ \\
\hline $\begin{array}{l}\text { Virginia Modeling, Analysis } \\
\text { and Simulation Center }\end{array}$ & VMASC & $\begin{array}{l}\text { Behavior of Small to } \\
\text { Medium Crowd in } \\
\text { Military Operations }\end{array}$ & $\begin{array}{l}\text { Military Train- } \\
\text { ing }\end{array}$ \\
\hline $\begin{array}{l}\text { Center for Human Modeling } \\
\text { and Simulation at University } \\
\text { of Pennsylvania }\end{array}$ & CHMS@UPenn & $\begin{array}{l}\text { Behavior of Small to } \\
\text { Medium Crowd }\end{array}$ & $\begin{array}{lr}\text { Military } & \text { Train- } \\
\text { ing, } & \text { General } \\
\text { Purpose } & \end{array}$ \\
\hline
\end{tabular}

Opinion Dynamics [Stauffer 2005] and Self-categorization Theory [Turner et al. 1989] etc, have been established to explain certain long-term crowd phenomena. These theories are usually in the form of some general observations and rules accompanied by a set of assumptions. Only until recently, modeling and simulation techniques have been introduced in this area, where the focus is on the development of computational models for existing social theories. 
A typical example is the series of models of opinion dynamics developed by Deffuant. The models have been designed to explore how extreme ideologies are formed and spread among the individuals in a crowd [Deffuant 2006], in particular how marginal extreme opinions become the norm in large parts of a population over a long period of time. The important modeling factors include the social network among the individuals, the evolution strategies of an individual's opinion and the learning processes of the individuals. A population is modelled as a network of interacting agents, and each agent can be simply characterized by its (continuous) opinion value and its uncertainty [Deffuant et al. 2002]. The uncertainty value is introduced to reflect the degree of willingness that an agent want to change its opinion. A small uncertainty value means that an agent is unlikely to change its opinion, and it also implies a higher probability that the agent will influence other agents' opinions and uncertainties.

The key of these models is the mathematical equations governing the evolution strategies of an individual's opinion. Although these models have been tested on simulated populations consisting of hundreds of people, as pointed out by Deffuant in [Deffuant 2006], the results are very sensitive to contingent mathematical choices of the parameters. Therefore, the models are quite restricted to the specific situations being considered. In addition, the behavioral factors being considered in these models are limited to some highly simplified social characteristics such as opinion and social network. Many important behavioral factors such as physical factors and psychological factors are omitted. An individuals' decision making process is simply reduced to a mathematical equation on the evolution strategy of opinion.

Another interesting work [Salzarulo 2006] is the meta-contrast model proposed by Salzarulo, which also investigate how people's opinions are affected by various factors based on the self-categorization theory. The meta-contrast principle contends that people tend to maximize the ratio of intergroup differences to intragroup differences, and as a result, different groups may emerge through the interactions among the individuals. In his model, an individual is modeled as an agent with a continuous opinion. An agent may change its opinion through the interaction with other individuals according to the meta-contrast principle. Salzarulo's model examines the impacts of several network interactions on the evolvement of people's opinions, and indicate that consensus, polarization or extremism are possible outcomes, even without explicit introduction of extremists as Deffuant's model does. Salzarulo's model suffers from the similar problems and limitations as we mentioned on Deffuant's models. For instance, the choice of $d\left(x_{i}, x_{j}\right)$ (the Euclidean distance between two opinions) and $\mu_{p}(x)$ (a fuzzy membership function of opinion $x$ to a prototype $p$ ) seems to be quite arbitrary, which may significantly affect the simulation result.

There are also some work that aim to explain how some plausible assumptions about individual action could yield certain properties that are observable at macro level [Miller and Page 2007; Gilbert 2007], for example, how neighborhoods are formed and may evolve in a community, how some social movements like 'punks' may be modelled, etc. These work tackle such problems from a high and abstract level, and are heavily influenced by research in game theory, complex adaptive systems, etc. The proposed generic models may help researchers do some simple 
experiments at abstract level, and also provide insights on the link between microlevel action and macro level properties. However, these models are often oversimplified without considering many factors in real-life situations. For instance, domain knowledge is generally abstracted away from these models.

In general, the existing work on the modeling and simulation of long-term crowd dynamics mainly concerns the statistical trend of some high-level aspects of a crowd (e.g., opinion) instead of a detailed description of how an individual in a crowd will react to certain events in more complex scenarios (e.g., how to move in a crowded shopping area when a fire breaks out). Thus, the proposed crowd models are limited to some high level operational studies, and are mainly of interest to social scientists rather than computer scientists and researchers in other areas. Execution efficiency and scalability are not the major concerns for these modeling and simulation efforts. These models are not flexible and extensible as they are usually specific to certain situations and their results are very sensitive to some chosen parameters. Currently, the majority of research and development efforts in crowd modeling and simulation is on short-term crowd phenomena, especially for small-to-medium sized crowds. This paper therefore follows this trend and emphasizes on the work of short-term crowd phenomena.

\subsection{Modeling and Simulation of Short-term Phenomena of Large Crowds}

The most representative approach of modeling crowds formed by tens of thousands of people is the flow-based approach. Unlike other approaches, the flow-based approach treats the crowd as a whole or continuum without considering the crowd's components such as individuals or groups. Typically, with the flow-based approach, vector fields are used to represent the impact of various environmental factors on the movement of the crowd, and the movement of a crowd is described using some differential equations. To form up these vector fields and differential equations, certain hypothesis and statistical assumptions are needed, whose validity are often debatable.

Recent research on flow-based approach started to consider the intelligence of crowd flows [Hughes 2003]. Researchers distinguish crowd flows from a classical fluid model by considering a crowd's capacity of thinking. The research of "thinking fluids" attempts to include factors like pedestrians' moving tendency in a crowd (e.g., avoiding extreme density), according to the observations of behavioral sciences. However, in general, the flow-based approach focuses mainly on the movement of the crowd, and other behavioral factors are greatly simplified or largely omitted.

A practical application of flow-based approach is the EVACNET4 software developed by Thomas M. Kisko and his colleagues at University of Florida [Kisko et al. 1998]. EVACNET4 provides modelers with a tool to build simulation models for complex building evacuations. In these models, the environment is abstracted into a network representation, which consists of nodes and arcs. The nodes represent the physical structures, such as rooms, stairs, and hallways. The node capacity is determined by the number of occupants the structure may accommodate. The arcs represent the passageways for the occupants' flow. Similarly, the arc capacity needs to be determined to limit the amount of human traffic that can traverse the passageway in certain time period. Another parameter, the traversal time, denotes the time an occupant flow takes to traverse the arc. A flow-based model constructed 
using EVACNET4 then relies on a specialized algorithm, used in solving linear programming problems with network structure, to generate optimal evacuation plan. Results can be used to determine the shortest evacuation time based on the network structure and corresponding physical constraints.

EVACNET4 offers great flexibilities in terms of the architectures and types of buildings. However, as a flow-based approach, EVACNET4 does not model behavioral aspects of crowd besides movement. In addition, compared to other flow-based models such as the one developed by Hughes [Hughes 2003], the movement of the crowd in EVACNET4 is greatly simplified - no vector fields and differential equations are used, i.e., the speed of the crowd is treated as piece-wise constant. These features limit EVACNET4 essentially to civil planning.

Recently, some significant work has been done in the computer graphics and animation community on animating large-sized crowds in virtual environments. In general, these efforts focus on the movement rather than other behavioral aspects of a crowd. In [Chenney 2004], Stephen Chenney (CGG@UW) presents the Flow Tiles technique for representing and designing velocity fields for large flows, including crowd flow. Each flow tile defines a small, stationary region of velocity field. They can be pieced together to form large stationary fields and then used to drive various flows such as fluids and crowds. In terms of crowd modeling and simulation, the flow tiles technique aims at producing visual effects of crowd motion rather than studying the dynamics of crowd. The major tasks of applying this technique include dividing the virtual environment into flow tiles and defining velocity fields on these tiles. These are delicate jobs requiring considerable effort. Except for movement, the behavioral factors of a crowd are largely ignored. Nonetheless, this technique is suitable for digital entertainment in general.

In [Treuille et al. 2006], Treuille et al described Continuum Crowds, a realtime motion synthesis model for large crowds. The model is inspired by Hughes' work [Hughes 2003] in the sense that it uses a similar potential function to guide crowds towards their goals. The model unifies global path planning and local collision avoidance into a single optimization framework. The model is based on a set of assumptions on the movement of a crowd, in particular, its global path planning assumes global knowledge of the environment which is often an unrealistic assumption. Nonetheless, the model is able to generate visually realistic movement for thousands of characters in near real-time. A research group at the VRLab of the Swiss Federal Institute of Technology (VR@EPFL) also work on the real-time animation of large crowd. Their work also focuses on the path planning for the crowd. Instead of relying on some predefined velocity fields, this work divides the environment into regions of varying interests. Then, for different regions, different levels of details are used for path planning and rendering. Experimental results show that this approach is able to generate near real-time animation for up to 10,000 virtual characters [Morini et al. 2007].

\subsection{Modeling and Simulation of Short-term Phenomena of Small-to-Medium Crowds}

Most research and development work on crowd modeling and simulations are around the short-term phenomena of small-to-medium crowds with tens to hundreds of individuals. This is mainly determined by the uses of crowd models in practice. Existing work in this area can be further divided by the approaches used for modeling 
crowds, which mainly include the entity-based approach and the agent-based approach. The former approach mainly denotes physicists' efforts of analyzing crowd dynamics using physical theories. The latter represents the main trend of research efforts in computer science and engineering domains. As we have pointed out in Section 2.1.1, sometimes the difference between an entity-based model and an agentbased model is not so clear. However, in general, they have different treatment of individuals in a crowd. That is, individuals in an entity-based model are largely modelled as homogeneous entities like physical particles, which is in contrast to the treatment of individuals in agent-based models where more complex decision making process as well as human behavioral factors are considered.

3.3.1 Entity-based Approach. In recent years, a number of physicists have employed physical and mathematical methods in the analysis of complex social phenomena. The study of crowd motion using basic physical theories and analytic models of individual pedestrians has been well established. The basic feature of these models is to handle a crowd at individual level and treat individuals as homogenous entities whose movement are governed by similar physical laws on physical particles (see Figure 4). These models are often called particle system models. The major challenge in designing such models is to properly (and often creatively) design the forces or potential fields to represent the impact of various behavioral and environmental factors on the movement of the individuals.

Dirk Helbing at Dresden University of Technology (Germany) has proposed a model based on physics and socio-psychological forces to describe the pedestrian behaviors in panic situations [Helbing et al. 2000]. The model relies on a quantitative analysis of panic, which is described by distance, velocity, mass and forces. With Helbing's model, individuals in a crowd are represented as particles with associated mass and velocity. The model assumes a mixture of physical and sociopsychological forces influencing the movement of an individual in a crowd.

More specifically, each of the entities represented by a particle $i$ of mass $m_{i}$ has a predefined desired speed $v_{i}^{0}$ in a certain direction $e_{i}^{0}$. The entity tends to adapt its actual velocity $v_{i}$ with a certain time interval $\tau_{i}$ towards the desired speed and direction. This corresponds to the first term on the right-hand side (RHS) of Equation 1. Simultaneously, the entity tries to keep a distance independent of velocity from other entities $j$ and walls $w$ controlled by interaction forces $f_{i j}$ and $f_{i w}$ respectively. This corresponds to the second and third terms on the RHS of Equation 1 . The change of velocity at time $t$ for each entity $i$ is mathematically formulated as in Equation 1 below:

$$
m_{i} \frac{d v_{i}}{d t}=m_{i} \frac{v_{i}^{0}(t) e_{i}^{0}(t)-v_{i}(t)}{\tau_{i}}+\sum_{j(\neq i)} f_{i j}+\sum_{w} f_{i w}
$$

The interaction force of $f_{i j}$ between entities is further composed of three type of social forces. The first one is a repulsive interaction force to model the psychological tendency of two pedestrians to stay away from each other. The second one is a body force to counteract body compression and the last one is a sliding friction force to impede relative tangential motion. The interaction force of $f_{i w}$ with walls is treated analogously with three forces involved. The mathematical details for formulating the interaction forces are available in [Helbing et al. 2000]. 
Helbing's model was tested with some simulation studies on crowd movement in a 2-D environment. Since the objective of these studies are on the emerging movement patterns of the crowd, each individual is simply represented by a dot. The simulation considers various evacuation situations, such as jamming at a congested exit, passing through a corridor and leaving a smoky room. The results are able to demonstrate some interesting behavior patterns (e.g., arch-like blockings of exit), crowding effect (e.g., faster-is-slower) and even optimal escape strategy (e.g., mixture of individualistic and herding behavior) during emergency situation. However, treating the individuals as homogeneous particles are not adequate to model the psychological and social factors of a human being, such as family ties, personalities, etc. These factors may greatly affect the movement of the individuals as well as the global patterns of the crowd. In addition, although various social forces have been introduced to model the effect of various urges (e.g., the psychological tendency of two pedestrians to stay away from each other) on an individual's movement, defining the exact magnitude of such forces requires much trials for the end results to look plausible. After all, these simulated social forces and the real physical forces on a human body are in two different domains. The computational cost of the model is generally high due to the quadratic complexity of force computation.

Helbing's model is extensible in the sense that it allows various behavioral factors to be modeled as some generalized forces. For example, Braun et al extends Helbing's model by introducing different personalities as well as group characteristics in their particle system model [Braun et al. 2005]. The extended work characterizes different individuals by a list of parameters including personal ID, family ID, dependence level, altruism level, and desired speed. These parameters may be used to model different persons. For example, a small value of the desired velocity and a high dependence level are used to represent a disabled person or a small child.

Burstedde et al at Institute of Theoretical Physics at University of Cologne (ITP@UC) have attempted to simulate pedestrian traffic using the particle system approach and a two-dimensional cellular automata model [Burstedde et al. 2001], which is a representative work among the research work of traffic flows by physicists. In the context of their approach, each individual is denoted by a particle and the space in which pedestrians move is abstracted as a grid of cells. The approach adopts a model with simple design which does not assume any intelligence of particles.

From a particle's perspective, each neighboring cell is associated with a transition probability to describe the particle's preference of motion direction according to the concepts of cellular automata model, as shown in Figure 5(A) and 5(B). A $3 \times 3$ transition matrix is constructed for each cell which defines the probabilities for a particle to move into neighboring cells or remain in its current cell. All particles of the same species share the same transition matrices. However, different species of particles may have different transition matrices (see [Burstedde et al. 2001] for details). The transition matrix of a particle can be affected by the static components of the environment, such as "emergency exit", and by the presence of other particles (pedestrians). The models are able to demonstrate a few collective phenomena of pedestrians, such as some emerging pattern in building evacuations (see Figure 5(C)). To apply this approach, considerable efforts are needed in divid- 
ing the environment into a grid of cells and in determining the transition matrices for these cells. The grid of cells and the transition matrices can be predetermined based on the situation of interest. The models are thus not flexible to handle different situations. However, compared to Helbing's model, these models have better performance in terms of execution efficiency and scalability due to the reduction of decision rules and environmental influences into simple lookup operations on the predefined transition matrices.

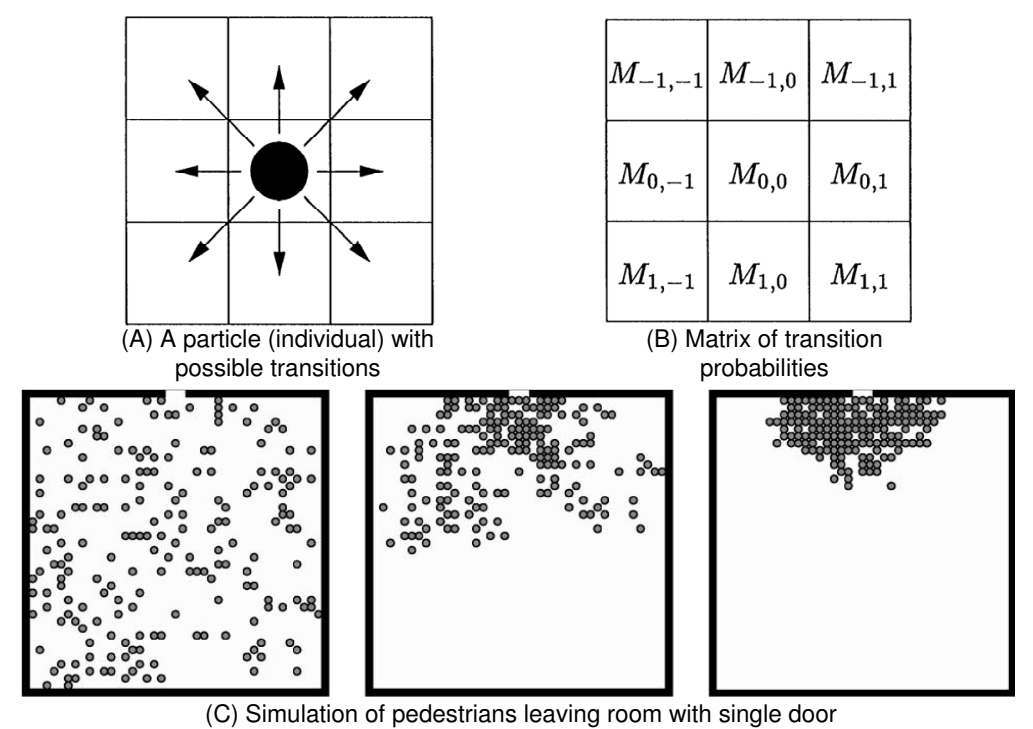

Fig. 5. Illustration of the cellular automata model. [Original figure was retrieved from reference [Burstedde et al. 2001].]

Michael Batty and his colleagues at the Center for Advanced Spatial Analysis at University College London (CASA@UCL) have done remarkable work in the modelling and simulation of the dynamics of pedestrian movements in spatial events like carnivals and street parades [Batty 2009; Batty et al. 2003]. The model that they have proposed is based largely on Helbing's model, which is built within a cellula automata structure. The environment consists of a (large) number of square cells. A pedestrian is represented as a particle that may move from its current cells to one of its eight neighboring cells in one simulation time step. An essential requirement of the model is the pre-computation of the relative accessibility of different attractions which make up the entire event with respect to the points where pedestrians enter the environment [Batty 2009].

The research group has adopted a nice modelling methodology to deal with the complex pedestrian dynamics in such events. That is, their model starts with a simple form of random walk. This simple random walk behavior is then further constrained by the geometry of the environment as well as economic and social preferences to reflect various factors in pedestrian movements. Finally, the movements of pedestrians are further constrained by certain safety control policies. In 
this sense, the model is generic that allows for new features to be added in to model different situations.

Currently, the model is mainly used for evaluating existing safety policies, i.e., it is not used for on-the-fly decision making in such events. Thus, execution efficiency and scalability are not major concerns of the model.

In summary, the models based on entity-based approach have successfully demonstrated some typical motion patterns of pedestrians in normal situations as well as in emergencies. Although considerable computing power are needed, simulation models based on this approach are relatively computationally inexpensive compared with the agent-based models. They are in general not adequate to investigate complex crowd behaviors, e.g., human decision-making process, and the social/psychological factors are either neglected or greatly simplified. Nonetheless, the particle system approach remains as a convenient and important tool for modeling and simulation of crowd behavior especially when the objective is to study the emerging movement patterns of a crowd under emergency situations. This is also manifested by the fact that most commercial crowd simulation software packages (e.g., EXODUS [Galea and Galparsoro 1993] and WalkSim [AG 2009]) for building planning and evacuation analysis are built from these classes of models. Interested readers may also refer to [Klugl and Rindsfuser 2007; Klupfel 2007] as well as the work done by Batty's group at CASA@UCL for some more recent crowd simulation applications with such models.

3.3.2 Agent-based Approach. The agent-based approach is currently the dominant and most active approach to crowd modeling and simulation particularly among computer scientists. In general, an agent-based crowd model consists of autonomous, interacting agents, with each agent representing an individual. Each agent has its own attributes and states representing various behavioral factors ranging from physical factors like moving speed to psychological/social factors like emotion, social ties, etc. An agent is also equipped with certain level of cognitive and reasoning capability such that it is able to sense its surroundings, make decisions based on its understanding of the current situation, and take actions accordingly which affect the environment as well as other agent's behavior.

The agent-based approach is the most natural way to model and simulate a crowd. Compared to other approaches, the agent-based approach gives a researcher tremendous freedom to incorporate various behavioral details into the crowd model. However, care must be taken for the modeling and simulation efforts to be effective. Existing work based on this approach may have different focuses with their different objectives. Nonetheless, an agent-based crowd modeling and simulation system usually needs to address three aspects: navigation, decision making, and animation.

Most existing work on crowd modeling and simulation focus on the movement of a crowd. Navigation is about how to drive the agents so that they can move around in the virtual environments. Usually, navigation is implemented as some path planning and steering algorithms. Decision making is to decide what an agent will do in the current situation (e.g., when an explosion occurs). Decision making can be implemented by some decision rules. These rules vary in their sophistication as how much information is considered in the agent decisions [Lebbink et al. 2005]. Even simple rules, for example the three local rules (cohesion, separation and align- 
ment) in Reynolds' boids model [Reynolds 1987] for simulating flocking or schooling behavior in birds or fishes, can generate complicated patterns. However, the decision rules are generally intuitive and specific to certain situations, thus defining the proper decision rules is often an art rather than a science. Animation is to visually show the results of a crowd simulation (i.e., the behavior of agents). It is especially important for digital media, entertainment, and interactive applications. Animation is also important for validation. That is, the SMEs (Subject Matter Experts) may make their judgement by comparing the visualized crowd behavior with their knowledge and experiences.

In the remainder of this section, we will discuss some recent research efforts in agent-based crowd modeling and simulation. We will discuss how the above three aspects are addressed in these work. Where possible, we will make comments on these work using our proposed evaluation criteria, based on the information that we have collected about these work. We also realize that there exists some commercial software that can be used to generate animations for crowd, such as MASSIVE [Massive Software 2009] and AI.Implant [Presagis 2009]. However, they are not included in the following discussion. There are three reasons. First, we have difficulty in getting the technical details of these software. Thus it is hard for us to make comments on them. Secondly, they are mainly for crowd animation rather than crowd simulation. While they provide excellent support for artists to visualize their design, they are not designed for computer scientists and engineers to investigate crowd behavior. Thirdly, path planning and decision making in these software actually adopt some common techniques such as A* algorithm [Dechter and Pearl 1985], and finite state machines (FSMs) [Sipser 2005]. We also noted some significant work done by researchers in the transportation and urban planning area, e.g., the work done by the Urban Planning Group at Eindhoven University of Technology [Arentze et al. 2006; Arentze and Timmermans 2009]. These works are not included in the following discussion since they describe agents' behavior from quite a different perspective, i.e., from an activity-based perspective, and therefore the focus of these work is on the rescheduling of activity-travel patterns of the agents.

Crowd Dynamics: Crowd Dynamics Pte Ltd aims at servicing police, event organizers and security forces. The company has developed a set of crowd modeling and simulation tools for different application. Though the company put its emphasis on the analysis of events involving large crowds [Crowd Dynamics Pte Ltd 2009b], it has also developed an agent-based modeling and simulation tool, called Legion, which is a collection of programs developed to analyze the dynamics of crowds. The core of these programs is a global path planning algorithm for agents. The path planning is formulated as a constrained optimization problem with the objective to minimize a general cost function. This path planning algorithm can be extended to accommodate navigational considerations provided that these considerations are properly reflected in the general cost function. A least effort algorithm is developed to solve this problem based on simulated annealing, which requires considerable computing time. Besides path planning, no other decision making is involved. Psychological and social factors are hardly considered. This tool is mainly used for operational analysis rather than interactive applications. Thus, execution efficiency 
and scalability are not of major concerns. The visualization of the simulation output is rather simple - the environment is represented by some 2D structures and connections, and each agent is represented by a simple $2 \mathrm{D}$ shape or simply a dot.

VRLab@EPFL: The Virtual Reality Lab at the Swiss Federal Institute of Technology (VRLab@EPFL) has done remarkable research in crowd modeling and simulation particularly in visualizing and animating virtual crowds. The technologies developed by VRLab have been applied in computer games, virtual heritage applications or training systems. A number of crowd simulations have been produced in various general virtual environments such as urban areas and transportation systems etc [de Heras Ciechomski et al. 2005; Ulicny and Thalmann 2001; Maim et al. 2007; Yersin et al. 2009]. The crowd modeling system adopts an agent-based framework called ViCrowd [Musse and Thalmann 2001; Yersin et al. 2005]. One key feature of ViCrowd is that it adopts a hierarchical modeling structure where a crowd consists of various groups with each group consisting of some individuals. This feature allows ViCrowd to model different social groups such as family and friend group. Another key feature of ViCrowd is that it has a flexible behavior control mechanism which allows for different degrees of behavior autonomy. Users can thus generate scripted behavior, reactive behavior or guided behavior, depending on the requirements of different applications.

The agents are represented by 3D avatars with different appearances which are able to move around in a 3D virtual environment. For path planning and collision avoidance with static obstacles, each agent is equipped with some knowledge about the environment. This is first achieved by the declaration of all objects in the environment as well as the areas where agents can walk. Then, to facilitate proper movement and action of the crowd, the interest points (IPs, denoting the locations where crowd need to pass) and action points (APs, denoting the locations where the crowd must perform an action upon arrival) are specified. No sophisticated path planning is used - basically, the path followed by the crowd is specified using a predefined set of IPs and APs that are associated with the groups of agents.

To simulate group movement, the agents in the same group share the same list of APs/IPs. In the meantime, different Bézier curves are computed for each agent in the same group so that they may take different paths while moving as a group. Then, by keeping at similar walking speed for the agents in the same group, flocking behavior can be easily achieved.

As a behavior authorizing tool for crowd animation, ViCrowd provides three types of behaviors with different degree of autonomy, namely, programmed behaviors, reactive behaviors, and guided behaviors. A script language is developed in ViCrowd for a user to program specific behaviors. Basically, it allows a user to defined where the crowd should move and what to do at some locations in terms of a list of IPs/APs. The reactive behavior allows an agent to react to the events depending on its current states. The decision making process is modeled using hierarchical finite state machines (FSMs). A major problem with this approach is that the number of state transitions may increase quickly as more behaviors are included. To accommodate this problem, some low level behaviors are predefined as basic building blocks such as flocking, following, attraction, splitting, etc, and 
the FSMs are defined only on some high level behaviors. Guided behavior provides external control that makes it possible for the crowd behavior to be controlled by a user or an external application.

ViCrowd provides good interface for a user to add in more behaviors to cater for a specific applications. In this sense, it is flexible and extensible. However, it is mainly a behavior authoring tool for crowd animation rather than a tool to investigate crowd behavior. For example, it allows different emotional states of an agent to be modeled, such as "sad", "happy", etc. However, the emotional states of the agents are predefined. The emotional states are then used to control the posture and appearance of the agents. How the emotion of an agent may change and how the resulted emotion will affect the agent's decision making are thus ignored.

The virtual characters in VRLab's crowd animations have state-of-the-art appearances and varieties. The computational and rendering cost is high especially for large crowd. To support real-time applications, advanced level-of-detail techniques have been developed so that regions with different level of interest may use different approaches for path planning, collision avoidance, and rendering [Pettre et al. 2006; Pettre et al. 2007]. For example, agents in regions of low interest may take on very simple appearances and may not even need to do path planning and collision avoidance. As a result, the crowd animation engine of VRLab is able to generate real-time animation for up to $10,0003 \mathrm{D}$ virtual characters [Morini et al. 2007].

MRL@NYU: The research group at the Media Research Lab in New York University focuses on developing a comprehensive behavior model for animating virtual pedestrians [Shao and Terzopoulos 2005a; 2005b; 2006]. Their virtual pedestrians are expected to perform a rich variety of activities in large-scale synthetic urban spaces. The two major features of their work include: 1) a novel environmental model that supports the navigation of virtual pedestrians in a complex urban environment such as the original Pennsylvania Station in New York City; and 2) an efficient behavior model of autonomous pedestrian agents.

The navigational behavior of the pedestrian agents are determined by the path planning and collision avoidance mechanisms, which in turn depends heavily on the representation of the environment. In MRL@NYU's virtual environmental model [Shao and Terzopoulos 2005b], an urban environment is characterized using a hierarchical collection of maps including a topological map, the perception maps and the path maps. The topological map represents the topological structure between different parts of the environment using a graph where nodes correspond to environmental regions and edges represent accessibility between regions. The perception maps include a number of local grid maps that represent stationary objects (one for each region) and a global grid map that keeps track of the moving agents. These perception maps will be used by the perception module of an agent to assess the current situation, such as the number and location of neighboring agents and obstacles.

The path maps include a quad-tree map that supports global, long-range path planning, and a grid map that supports short-range path planning. $A^{*}$ algorithm is then applied on the quad-tree map to compute a rough path to a goal which consists of a list of key points leading to the goal. The detailed path between two 
points along the rough path is determined by applying $A^{*}$ algorithm on the grid map. This hierarchical path planning approach is more close to the way how a real pedestrian does path planning. In addition, it is very efficient. That is, the detailed path from point $\mathrm{A}$ to point $\mathrm{B}$ is computed only when the agent has reached point A. While the agent is moving from A to B following the detailed path, if something happens that makes the detailed path infeasible, only this detailed path needs to be recomputed. The rough path for the agent still remains unchanged. This is an important feature that can greatly enhance the execution efficiency as well as scalability. The experimental results show that the environmental model is able to support real-time simulation of about 1,400 pedestrian agents on a $2.8 \mathrm{GHz}$ Xeon PC with 1GB memory.

The autonomous agent model is heavily influenced by the work on artificial animals [Tu and Terzopoulos 1994] and cognitive modeling for intelligent characters [Funge et al. 1999]. To imitate a real pedestrian, an agent is equipped with the ability to perceive the environment around it, make decisions on what to do, and then take actions. To perceive the environment, an agent needs to query the previous mentioned environment maps and do some line-of-sight and range tests. One particular feature that has been implemented is that once a predefined number of nearby agents are detected, the sensing is terminated. This is to model the cognitive limits of human attention system. That is, at any given time, people usually pay attention only to a limited number of other people (usually those closest ones).

To address the challenge in modeling the variety of pedestrian behaviors, a hierarchical structure with a bottom-up strategy is adopted. That is, primitive reactive behaviors are first defined as basic building blocks, and more complex deliberative behaviors are then defined by these primitive behaviors controlled by an action selection mechanism.

The 3D appearances and low-level motor skills (e.g., standing, strolling, walking, running, etc) of the agents are implemented on DI-Guy [Boston Dynamics 2009], which is a commercial product. Six basic reactive behavior routines are then developed for initiating, terminating, and sequencing of the low-level motor skills, guided by sensory stimuli and internal percepts. Each of these six behavior routines is suitable for a different set of situations, such as cross collision, head-on collision, etc. (See [Shao and Terzopoulos 2005a] for details.), in a densely populated and highly dynamic urban environment.

More complex and deliberative navigational behaviors are implemented by an action selection mechanism. The action selection mechanism needs to decide when to initiate a proper basic reactive behavior and when to switch to another reactive behavior, based on an agent's perception and current states (such as physical, and psychological states). The selection mechanism is implemented by some decision rules based on some observations on pedestrian behavior. For instance, in real life, once oncoming traffic is encountered when people are moving in a narrow passageway, they will tend to form opposing lanes to maximize the throughput. This passageway navigation behavior can be implemented by a series of "if-then" selection rules: First, if an agent detects that its current direction is blocked by oncoming agents, it will search for a safe direction to get through. If the search is successful, it will move in the new direction; otherwise, it will slow down and 
proceed in the rightmost direction within a safety fan area.

The above hierarchical path planning and behavior control mechanisms are quite flexible to implement various pedestrian behaviors. Though the current work focuses on the navigational behavior, it is possible to incorporate more factors such as psychological and social factors into the action selection mechanisms. The hierarchical architecture also enhances the execution efficiency and scalability of the simulation system. Experimental results show that the computational load increases nearly linearly with the number of agents in the simulation.

VMASC: The research group led by Dr. Mckenzie at the Virginia Modeling Analysis and Simulation Center (VMASC) in Old Dominion University focuses on investigating psychologically-based real-time interactive crowd model for military simulation in urban settings [McKenzie et al. 2004; Nguyen et al. 2005; Petty et al. 2004]. Since their focus is not on crowd animation, they rely on some commercial products such as Forterra Systems' OLIVE (http://www.forterrainc.com/) for crowd animation as well as the basic navigation. Here we comment only on their psychologically-based behavior modeling framework.

To make the crowd behavior model flexible and extensible, a behavior hierarchy is proposed, which consists of a cognitive layer at the top and a physical layer at the bottom. Each layer is responsible for modeling different aspects of the crowd model. The cognitive layer is responsible for modeling the "mind" for the crowd. That is, it will receive stimuli from the physical layer, process the stimuli, and select an appropriate behavior for the physical model to carry out. The physical layer acts like the "body" of the crowd and is responsible for get stimuli from the environment and executing behaviors that are decided by the cognitive model.

The major design considerations of the crowd model thus include: 1) specifying stimuli; 2) defining the repertoire of behaviors; and 3) developing the computational model of the decision-making (i.e., behavior selection) process. It should be noted that VMASC's crowd model is targeted at military operations and thus is based on some psychological studies on military operations [Petty et al. 2003]. The stimuli and psychological factors are therefore closely related to military operations. For example, the stimuli that have been considered include "see nearest friend", "hear militia broadcasting on megaphones", "hear gunfire", "leader is shot", etc. In addition to some simple behaviors, more complex behaviors in the current implementation include "wandering", "fleeing", "building barricade", "throwing rocks", etc. Although other stimuli and behaviors could be added into the crowd model, this will involve significant amount of work as we will explain later.

To support real-time interactive simulation, the crowd cognitive model is focused at the group and crowd levels - no individual cognitive model is implemented. The assumption here is that a realistic crowd behavior can be achieved without modeling the cognitive processes of all the individuals (This assumption is certainly debatable and its validity may be largely determined by the specific application and scenario). Thus, the mental states of the crowd and of the group have been simplified to a single state called the crowd aggression level (CAL) and the group aggression level (GAL), respectively. Again, this simplification also reflects the objective of the crowd simulation - investigating what makes crowds turn violent. Certainly, for other applications, more mental states are needed (for example to capture different 
emotions, such as jealousy level, fear level, etc).

The decision making process is essentially some simple lookup operations on some predefined tables. For example, a Mood-Behavior table is constructed which consists of multiple rows of <aggression level, behavior $>$ pairs. This table is used by the cognitive model to determine the appropriate behavior given the current aggression level of group or crowd. For example, if the current GAL is "Curious", then the corresponding behavior will be "Stare At". Other tables are also constructed to determine, for example, the GAL given the current value of certain stimuli.

It should be mentioned that the crowd model is rather simplified and specific to the applications of interest. It also caters for real-time interactive applications. However, as the decision making process is tightly coupled with the predefined tables and stimuli, adding in new behavioral factors will result in significant change to the simulation model.

CHMS@UPenn: Researchers in the Center for Human Modeling and Simulation at University of Pennsylvania have worked on improving the realism of human behavior models by integrating a set of psychological factors into a unified behavior architecture [Silverman et al. 2006; Silverman et al. 2006; Pelechano et al. 2007; 2008].

Like most agent-based behavior models, an agent in CHMS's work also follows the "sense-decide-act" cycle to respond to various events in the environment. A major contribution of the group in crowd modeling and simulation is that they have modelled the effects of various psychological factors on human decision making.

As illustrated by numerous studies (See [Loewenstein and Lerner 2003] for a quick reference), psychological factors such as emotion play important role in human decision making, which will significantly affect how a human behaves in various situations. For example, in a fire evacuation scenario, people that have not been properly trained are likely to feel stressed. This may seriously affect their capability in making the right decision due to time pressure. On the other hand, a well-trained person like a fire fighter is able to deal much better with the dynamically changing environment and time pressure.

To model the effect of psychological factors on decision making, PMFServ has been developed by the group [Silverman et al. 2006]. PMFServ is implemented as a large library (around 500) of Performance Moderator Functions (PMFs). Essentially, the PMFs take the form of some nonlinear functions that relate certain psychological factors with certain physiological factors. The PMFs are represented using a common mathematical framework and organized into four sub-modules with a layered architecture (from bottom to top layer): Stress PMFs, Perception PMFs, Emotion PMFs, and Decision PMFs. The modules address different aspects of human decision making process. Each module consists of quite a number of different PMFs. Nonetheless, the principal feature of PMFServ is a model of decision-making, based on emotional subjective utility constrained by stress and physiology, which is inspired by Ortony's OCC model [Ortony et al. 1988]. The general idea of the OCC model is that an agent possesses three hierarchical trees that describe its Goals for Action, Standards for Behavior, and Preferences for People, Objects, and Situations. An action can be represented by a series of successes or failures on the nodes of these three trees. Each sub-goal is given a weight that 
describes how much it contributes to its parent node. An action's emotional utility can be computed by some weighted sum operation on these trees. The action with the highest utility value will be chosen and executed.

The PMFServ is flexible in the sense that it allows a modeler to choose the proper PMFs to suit his/her specific application. However, determining which PMFs to use is not an easy task. In particular, determining the value of various parameters of these PMFs and the value of the weights of sub-goals for action selection needs considerable efforts, and depends heavily on the specific applications and situations of interest.

PMFServ essentially models an agent's mind, that is, it determines what to do. For an agent to be fully functional in a virtual environment, MACES has been developed by the group. MACES mainly deals with the locomotion of the agents in virtual environments [Pelechano et al. 2005; Pelechano and Badler 2006], which is used to implement and illustrate an agent's body. It computes agent's navigation at two levels. The high level module is a path planning module used to determine a rough path, e.g., the sequence of rooms towards an exit in an evacuation scenario; whereas the low level module is used to determine the detailed movement along the rough path, e.g, the local movement with each room.

The local motion within each room is based on Helbing's social force model [Helbing et al. 2000] that treats an agent as a physical particle subject to some general forces. The unique feature of MACES lies in its high level path planning. For path planning, each agent is equipped with its own mental map which abstracts the geometry of the building and is represented by a graph, where nodes are the rooms and the arcs are the portals between rooms. The mental map of an agent will be expanded as the agent explores the environment and shares information with other agents in the crowd through a communication process.

Communication occurs only among agents in the same room. It includes information about the location of hazards that block possible paths, the direction within a room that have been explored, and where no exit was found. Limiting the communication among agents in a room helps to reduce the computational overhead. This localized communication can also be seen in real-life situations.

Different mental maps are also used to represent the different knowledge of different people. For example, staffs working in the building usually have complete knowledge about the building's internal structure, while visitors may have little knowledge of the building regarding the locations of the exits. The feature allows a modeler to assign different roles to the agents in the crowd, which will make the simulation result more realistic. For example, the experimental results from MACES show that only a relatively small percentage of trained leaders (with building plan knowledge) yields evacuation rates comparable to the case in which everyone is trained. This observation is interesting and also intuitive.

In general, the research group at CHMS has done remarkable work in modeling the psychological aspects of human behavior for simulation. Their PMFServ model has been successfully used in simulating various crowd scenarios such as terrorist ambush of a school bus, crowd protesting/rioting at a roadblock, building evacuation, etc. However, the computational overhead may increase quickly as the number of PMFs increases to accommodate more complex situations. Therefore, a 
critical decision is to determine what PMFs to use for a specific application. This is especially important for situations involving a large crowd.

\subsection{Summary}

In this section, we have discussed some influential existing work in crowd modeling and simulation. Figure 6 shows how the crowd models in these existing work are distributed in a two dimensional plane defined by the time-scale and the size of the crowd. It can be clearly seen from this figure that the majority of the existing work are on the investigation of the short-term phenomena of small to medium sized crowds. This reflects the fact that most daily life situations involve small to medium sized crowd and people are interested in how a crowd will react to certain events.

Another thing that we can find from Figure 6 is the dominance of agent-based approach in crowd modeling and simulation. Due the increase in computing power, researchers tend to adopt a microscopic approach. That is, more and more behavioral factors of the individuals are considered in the agent models of the individuals. Individuals are treated as autonomous agents that are equipped with human-like functional modules including perception, cognition, decision-making, and action, etc. In this regard, we want to emphasize two points. First, including too much details in the agent model may not necessarily give more realistic results. An interesting fact about crowd is that crowd phenomena are in general robust to some minor differences or changes at the individual level. Secondly, the relations, particularly the social relations among the individuals, must be emphasized and properly modeled. As a complex adaptive system, crowd's behavior may not be understood simply by zooming into the individuals no matter how many factors we introduce in the individual behavior model. Theories and observations from social psychology may play a key role in bridging the individual behavior and the crowd phenomena.

Table II summarizes the major features of some agent-based crowd modeling and simulation work that we have discussed. We hope to provide readers with some quick reference about these work regarding the major behavioral factors that have been considered and the runtime performance. Although different path planning and decision making mechanisms may be used in these work, we can still identify some common features of these mechanisms. First, a hierarchical structure is widely used in path planning and decision making, which often makes path planning/decision making process very efficient. In fact, the hierarchical structure reflects the divide-and-conquer strategy that are often used by humans to make decisions in everyday situations. Second, the decision making processes are often implemented as certain forms of rule-based systems, for instance, (hierarchical) finite state machines. Despite some latest attempts [Lerner et al. 2007; Lee et al. 2007; Bandini et al. 2007], generally speaking, defining the proper rules to realize certain behaviors is often an art rather than a science. In particular, determining the parameters involved in these rules is largely a trial and error process that requires a lot of effort. Moreover, the selected rules and parameters are often tightly coupled with a specific situation. This makes them hardly applicable to other situations.

To resolve the above problem and also to make the decision making process more human-like, a promising alternative is the Recognition-Primed Decision (RPD) the- 


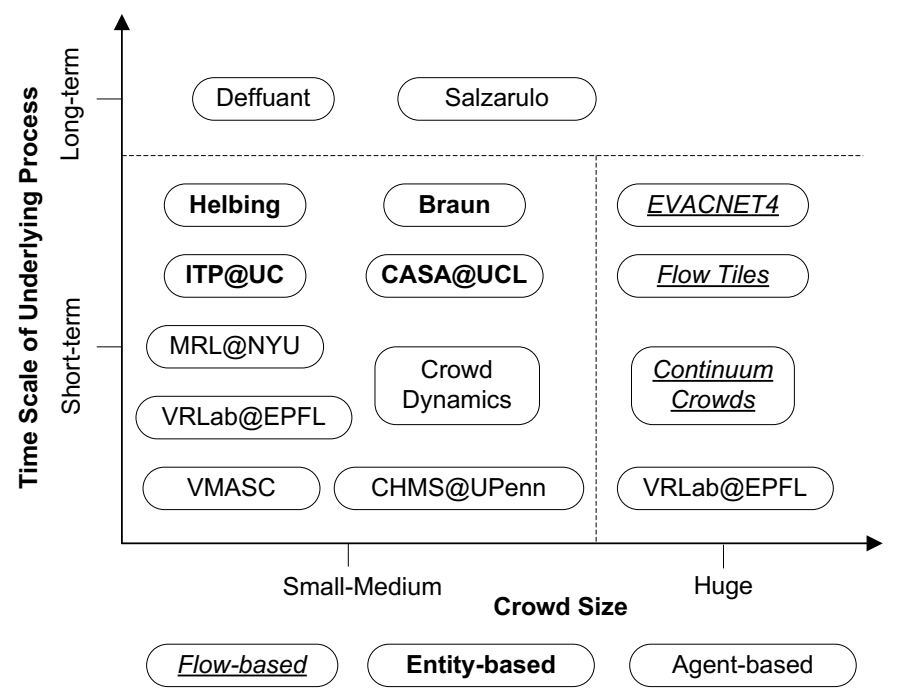

Fig. 6. Distribution of crowd models and simulation systems.

Table II. Comparison Among Existing Agent-based Crowd Models and Simulation Systems

\begin{tabular}{|c|c|c|c|c|c|}
\hline & \multicolumn{3}{|c|}{ Behavioral Factors } & \multicolumn{2}{|c|}{$\begin{array}{l}\text { Evaluation on Runtime Perfor- } \\
\text { mance }\end{array}$} \\
\hline $\begin{array}{l}\text { Research } \\
\text { Group }\end{array}$ & $\begin{array}{l}\text { Physical } \\
\text { Factors }\end{array}$ & $\begin{array}{l}\text { Social } \\
\text { Factors }\end{array}$ & $\begin{array}{l}\text { Psychological } \\
\text { Factors }\end{array}$ & $\begin{array}{l}\text { Number of Agents } \\
\text { (Scalability) }\end{array}$ & $\begin{array}{l}\text { Execution } \\
\text { Efficiency }\end{array}$ \\
\hline Deffuant & No & Limited & Limited & hundreds & Not applicable \\
\hline Salzarulo & No & Limited & Limited & hundreds & Not applicable \\
\hline $\begin{array}{l}\text { Crowd Dynam- } \\
\text { ics }\end{array}$ & Yes & $\begin{array}{l}\text { Almost } \\
\text { no }\end{array}$ & Almost no & Thousands & real-time \\
\hline VRLab@EPFL & Yes & Limited & Limited & $\begin{array}{l}\text { Tens to Tens of } \\
\text { Thousands }\end{array}$ & real-time \\
\hline MRL@NYU & Yes & Limited & Limited & Less than 1,400 & real-time \\
\hline VMASC & Yes & $\begin{array}{l}\text { Almost } \\
\text { no }\end{array}$ & Yes & Not available & Not available \\
\hline CHMS@UPenn & Yes & Limited & Yes & Less than 1,800 & real-time \\
\hline
\end{tabular}

ory [Klein 1998] advocated by the naturalistic decision making community. RPD is believed to be a more realistic account of how human make decisions in everyday situations (particularly, time-critical situations), which emphasizes the role of experiences and knowledge in decision making. Recently, RPD based models have been used in human-agent collaboration [Fan et al. 2005] and military simulations [Ting and Zhou 2008].

\section{DISCUSSIONS}

Although some significant work has been done in crowd modeling and simulation, it is still a relatively young research area. Many open research issues are still very much in flux due to the complexity of individual and crowd behaviors. In the section, we single out three issues that we observed recurring in the existing models ACM Journal Name, Vol. V, No. N, Month 20YY. 
and systems. We believe that these issues are the important open problems to be tackled in order to advance the research and practice in crowd modeling and simulation. We also feel that these issues are either inadequately treated or largely ignored in the existing work. Here, we give our insights into these problems.

(1) Level of detail of simulation model

A decision of primary importance that a modeler needs to make is the level of detail of the crowd model. To make this decision, a modeler must consider issues like whether individual behavior needs to be modelled or the crowd can be treated as a whole, whether individuals in the crowd can be treated as homogenous or heterogenous entities, whether the psychological and social factors need to be consider or not. Very often, answers to these questions are application-dependent and subject to the goal of the simulation study. The existing models are largely at two extreme levels - either model each individual as an autonomous agent equipped with some human-like behavior modules such as locomotion, perception and decision-making (e.g., the work done by CHMS@UPenn), or treat the crowd as a collection of homogeneous particles with limited or no cognitive features (e.g., Helbing's model and the work done by ITP@UC).

With the fast development of computing technology, there seems to be a trend in crowd simulation to model each individual as some kind of intelligent agent with attempts to incorporate more and more social and psychological factors into the agent behavior model. However, we believe that the shortcomings that the existing models have in common are the absence of modeling the social group process and its impact on human behavior. As social animals, people's behavior can be influenced by the social group they are associated with. For instance, in an emergency evacuation situation, a person's decision on whether and when to evacuate may influence and be influenced by the decisions of other persons in the same group. It is also natural for a father to first locate and gather all his family members before evacuating himself from the danger.

As to social group relation, a family can be regarded as a kind of fixed group bonded by the strong kinship relation. Some other kind of group relations may not be so strong and are thus dynamic. For example, groups of strangers may be formed along the way of evacuation since the danger they perceived may cause them to feel a common fate (threat) so that they re-identify themselves as a member of a group sharing the same goal. Thus, we believe group dynamics is an essential dimension that must be considered in crowd simulation. In this regard, the emergent norm theory [Turner and Killian 1987], the social attachment model [Mawson 2005], and social comparison theory [Festinger 1954] of social groups appear to be quite relevant. These theories help to combine the emphasis on the social psychology of an individual with an interest in macro features such as norms, values, status demands, leadership, and emergent beliefs. Recently, some researchers have attempted to incorporate some social theories into their agent-based crowd model. For example, Kaminka and his colleagues have developed a cognitive model based on social comparison theory of crowd behavior [Kaminka and Fridman 2006]. To simulate the effect of social ties on people's coping style in emergent situations, the research group at Nanyang Technological University has incorporated the social attachment theory into their agent behavior model [Luo et al. 2008]. 
(2) Validation and generalization

Model validation is one of the most difficult issues in crowd modeling and simulation. We discuss this issue here rather than in previous sections for two reasons. First, a model's validity heavily depends on the objectives of simulation study and the way the model is used. In this sense, this issue is generally orthogonal to crowd modeling techniques as well as those relatively well-defined evaluation criteria that we have proposed in Section 2.2. Second, despite its importance, progress in crowd model validation remains slow and limited, compared to the large number of crowd simulation models that have been developed.

The most effective approach to establishing model validity is to compare the simulation result of the model with the output data from the system being modelled. For example, for validation of a model of pedestrian behaviors, cameras could be deployed around an area of interest to capture data related to pedestrian movement. These recorded data are then used to validate and calibrate the results of the simulated movement of the pedestrians [Batty et al. 2003; Seyfried et al. 2005; Antonini et al. 2006; Fang et al. 2008].

However, as admitted by many researchers, a major difficulty in applying this results validation approach lies in the insufficiency of detailed data, especially for agent-based crowd models that take into account the decision making process and behavioral factors of individuals [Aguirre 2005; Batty et al. 2003; Klugl 2008; Pelechano et al. 2008; Galea 1998]. For example, a simulation of emergency evacuation has three distinct analytical dimensions: the physical environment from which people evacuate, the managerial policies and controls deployed at evacuation, and the psychological and social organizational characteristics impacting the persons who participate in the evacuation [Aguirre 2005]. Although it is not difficult to model the physical environment and managerial policies and controls for an existing system, modeling the psychological and social aspects of evacuation in the given system must first solve the problem of insufficient data.

For some relatively simple tasks involving only a small number of individuals, data could be collected in a controlled experimental environment, such as in [Seyfried et al. 2005]. However, data collected in such laboratory experiments are generally not sufficient to capture the rich crowd behavior in real life situations. Moreover, for most laboratory experiments, the participants often appear emotionally relaxed, which may make them behave differently as in real-life situations such as fire evacuation.

Another common approach to model validation is to check the face validity of the model, i.e., to check whether the simulation results of the model are consistent with the perceived system behavior by some subject matter experts (SMEs). This approach has been adopted by many existing crowd simulation systems [Helbing et al. 2000; Nguyen et al. 2005]. However, the SMEs' judgements are largely determined by their knowledge and experiences with past similar (or even quite different) emergency evacuations, which may have serious bias in the validation results. Recently, Pelechano et al proposed to use the level of presence achieved by a human in a virtual environment as a metric for validating crowd behavior [Pelechano et al. 2008]. Different from the computer animation community who has been primarily concerned with external crowd motion features, they focus on some internal or ego- 
centric features that may significantly influence the level of presence of a human in a virtual environment.

In [Klugl 2008], Klügl argued that a combination of different validation techniques should be used for validating agent-based simulations based on observations of some characteristics of multi-agent systems and the limitations of existing validation techniques. He proposed a validation process that combines different validation techniques.

We believe that some well-tested basic components (e.g., locomotion, perception, psychological characteristics of individual, social group dynamics etc) of crowd behavior must be built before a credible and comprehensive crowd simulation model can be achieved. In this regard, the work done by CHMS@UPenn in constructing a large number of physiologically and psychologically based PMFs (Performance Moderator Functions) is a good step forward. For evaluating pedestrian steering behaviors, Singh et al proposed a benchmark suite that consists of 38 testing scenarios with different level of complexity [Singh et al. 2009].

The model validation problem naturally brings about the generalization issue of the crowd simulation model. That is, whether a valid crowd model in a specific case can be applied to other similar cases. The generalization property is certainly desirable for a crowd simulation model to be predictive for different testing conditions. The simulation models in the existing work are usually built for a specific case under specific condition. The generalization issue has not yet been studied for these models.

(3) Interoperability and composability

The interoperability and composability issue is largely ignored by the existing research. Although there exist quite a number of crowd simulation models, these models can hardly work with each other: they may operate at different levels of abstraction. This makes the communication between different models difficult. In a typical crowd simulation, the crowd model needs to pass along various information about the actions of individuals and also needs to understand the events in the simulated world so that the individuals can determine how to respond to these events. However, different crowd models may use different models of perception and action, and may respond to events at different level of abstraction. For example, some models may work at a higher level of abstraction, thus are able to respond to events like "Being Insulted" and are able to produce abstract directives like "Show Anger"; while some models may work at a lower level of abstraction, thus are able to respond to events like "Being Pushed", and are able to produce actions like "Shouting Get-Out".

Research in interoperable crowd simulation models is still rare. A good preliminary work has been done in the SCALE-UP project [McDonald et al. 2006] where a five-level language has been proposed to describe agent behavior. This language aims at supporting the communication between behavior models at different levels of abstraction. Such a language may form the basis for a message standard that enhances the interoperability and composability amongst different human behavior models in crowd simulation. 


\section{CONCLUSIONS}

Recent years have witnessed a huge interest in crowd modeling and simulation from researchers, entertainment industries, and government agencies, with many papers being published and many projects being conducted. However, crowd modeling and simulation is still a relatively young research area. Technologies for crowd modeling and simulation are far from mature.

Crowd behaviors are complex. How a crowd will behave in certain situations often depends on many factors for different domains, such as physical factors, psychological factors, and social factors, etc. A researcher in crowd modeling and simulation thus faces many challenges as to which approach to use to model the crowd behavior, which behavior factors to consider, what level of detail to work on, which decision making mechanism to use, etc, given the objectives of the project and constraints of available resources. In this paper, we give an overview of the research and development of this area. We first classify existing work along two dimensions, namely, the size of the crowd and the time-scale of the crowd phenomenon of interest. This classification helps a researcher to clearly see how existing research and development efforts are distributed. Then from a modeler's point of view as well as a user's point of view, four evaluation criteria have been proposed, which will help a researcher to have a quick evaluation of the performance of the existing work and to decide whether the technologies in the existing work can be applied in his/her own project.

We have described some influential existing work in crowd modeling and simulation. Major features, performance as well as the technologies used in these work have been discussed. Base on our observation on the existing work, some open problems in the area have also been identified and discussed. We hope that these discussions provide the readers with useful information and insights on the stateof-the-art of the technologies in crowd modeling and simulation as well as future research directions.

\section{REFERENCES}

AG, S. S. 2009. Simwalk transport white paper. http://www.simwalk.com/simwalk_transport/ whitepapers/simwalk_trans_09_e.pdf.

Aguirre, B. E. 2005. Emergency evacuation, panic, and social psychology: Comment on understanding mass panic and other collective responses to threat and disaster. Psychiatry 68, 2.

Antonini, G., Bierlaire, M., And Weber, M. 2006. Discrete choice models of pedestrian walking behavior. Transportation Research Part B 40, 667-687.

Arentze, T. And Timmermans, H. 2009. A need-based model of multi-day, multi-party activity generation. Transportation Research Part B 43, 251-265.

Arentze, T., Timmermans, H., Janssens, D., and Wets, G. 2006. Modeling short-term dynamics in activity-travel patterns: from aurora to feathers. In Proceedings of the Innovations in Travel Demand Modeling Conference (21-23). Austin, Texas, 71-77.

Bandini, S., Federici, M. L., And Vizzari, G. 2007. Situated cellular agents approach to crowd modeling and simulation. Cybernetics and Systems 38, 7, 729-753.

BatTy, M. 2009. Agent-based pedestrian modelling. http://eprints.ucl.ac.uk/237/1/paper61. pdf.

Batty, M., Desyllas, J., And Duxbury, E. 2003. The discrete dynamics of small-scale spatial events: agent-based models of mobility in carnivals and street parades. International Journal of Geographical Information Science 17, 7 (October), 673-697.

Boston Dynamics. 2009. Di-guy. http://www.diguy.com/diguy/.

ACM Journal Name, Vol. V, No. N, Month 20YY. 
Braun, A., Bodmann, B. E. J., And Musse, S. R. 2005. Simulating virtual crowds in emergency situations. In Proceedings of the ACM symposium on Virtual reality software and technology (2005). 244-252.

Burstedde, C., Klauck, K., Schadschneider, A., and Zittartz, J. 2001. Simulation of pedestrian dynamics using a two-dimensional cellular automaton. Physica A: Statistical Mechanics and Its Applications 295, 3-4, 507-525.

Chenney, S. 2004. Flow tiles. Proceedings of the 2004 ACM SIGGRAPH/Eurographics symposium on computer animation, 233-242.

Crowd Dynamics Pte Ltd. 2009a. Crowd disasters. http://www.crowddynamics.com/Main/ Crowddisasters.html.

Crowd Dynamics Pte Ltd. 2009b. Crowd dynamics. http://www.crowddynamics.com/.

de Heras Ciechomski, P., Schertenleib, S., Maim, J., and Thalmann, D. 2005. Reviving the roman odeon of aphrodisias: Dynamic animation and variety control of crowds in virtual heritage. In Proceedings of the 11th International Conference on Virtual Systems and Multimedia. Ghent, Belgium.

Dechter, R. and Pearl, J. 1985. Generalized best-first search strategies and the optimality of a*. Journal of the ACM 32, 3 (July), 505-536.

Deffunant, G. 2006. Comparing extremism propagation patterns in continuous opinion models. Journal of Artificial Societies and Social Simulation 9, 3.

Deffunant, G., Amblard, F., Weisbuch, G., and Faure, T. 2002. How can extremism prevail? a study based on the relative agreement interaction model. Journal of Artificial Societies and Social Simulation 5, 4.

Fan, X., Sun, S., McNeese, M., And Yen, J. 2005. Extending recognition-primed decision model for human-agent collaboration. In Proceedings of the 4 th International Joint Conference on Autonomous Agents and Multi Agent Systems (AAMAS 2005). Utrecht University, Netherlands, 945-952.

FANG, Z., YuAn, J., WANG, Y., AND Lo, S. 2008. Survey of pedestrian movement and development of a crowd dynamics model. Fire Safety Journal 43, 459-465.

Festinger, L. 1954. A theory of social comparison processes. Human Relations 7, 2, 117-140.

Funge, J., Tu, X., And Terzopoulos, D. 1999. Cognitive modeling: knowledge, reasoning and planning for intelligent characters. In Proceedings of the 26th International Conference on Computer Graphics and Interactive Techniques (SIGGRAPH'99). Los Angeles, CA, USA, 2938.

GALEA, E. 1998. A general approach to validating evacuation models with an application to exodus. Journal of Fire Sciences 16, 6, 414-436.

Galea, E. R. and Galparsoro, J. M. P. 1993. Exodus: An evacuation model for mass transport vehicles. UK CAA Paper 93006 ISBN 086039 543X. http://fseg.gre.ac.uk/exodus.

Gilbert, N. 2007. A generic model of collectivities. Available at http://epubs.surrey.ac.uk/ cress/14.

Gratch, J. And Marsella, S. 2004. A domain-independent framework for modeling emotion. Journal of Cognitive Systems Research 5, 4, 269-306.

Helbing, D., Farkas, I., And Vicsek, T. 2000. Simulating dynamical features of escape panic. Letters to Nature 407, 487-490.

Hughes, R. L. 2003. The flow of human crowds. Annual Review on Fluid Mechanics 35, 169-182.

Kaminka, G. A. And Fridman, N. 2006. A cognitive model of crowd behavior based on social comparison theory. In Proceedings of the AAAI-2006 workshop on cognitive modeling and agent-based social simulation. Boston, MA.

Kisko, T. M., Francis, R. L., And Nobel, C. R. 1998. Evacnet4 user's guide. http://www.ise. ufl.edu/kisko/files/evacnet/EVAC4UG.HTM

Klein, G. 1998. Sources of Power: How People Make Decisions. MIT Press, Cambridge, Massachusetts. 
KLUGL, F. 2008. A validation methodology for agent-based simulations. In Proceedings of the 23rd Annual ACM Symposium on Applied Computing (SAC'08) (16-20). Fortaleza, Ceara, Brazil, $39-43$.

Klugl, F. And Rindsfuser, G. 2007. Large-scale agent-based pedestrian simulation. Lecture Notes in Computer Science 4687, 145-156.

Klupfel, H. 2007. The simulation of crowds at very large events. In Traffic and Granular Flow'05. Springer, Berlin, 341-346.

Lebbink, H. J., Witteman, C. L. M., And Meyer, J.-J. C. 2005. Tutorial on agent-based modeling and simulation. In Proceedings of the 2005 Winter Simulation Conference. Orlando, FL, 2-15.

Lee, K. H., Choi, M. G., Hong, Q., And Lee, J. 2007. Group behavior from video: A data-driven approach to crowd simulation. In Proceedings of the 2007 ACM SIGGRAPH/Eurographics Symposium on Computer Animation (SCA 2007). San Diego, CA, 109-118.

Lerner, A., Chrysanthou, Y., And Lischinski, D. 2007. Crowds by example. Computer Graphics Forum 26, 3, 655-664.

Loewenstein, G. And Lerner, J. 2003. Handbook of Affective Science. Oxford University Press, Oxford, New York.

Luo, L., Zhou, S., Cai, W., Low, M. Y. H., Tian, F., Wang, Y., Xiao, X., and Chen, D. 2008. Agent-based human behavior modeling for crowd simulation. Computer Animation and Virtual Worlds 19, 3-4 (August), 271-281.

Maim, J., Haegler, S., Yersin, B., Muller, P., Thalmann, D., and Gool, L. V. 2007. Populating ancient pompeii with crowds of virtual romans. In Proceedings of the 8th International Symposium on Virtual Reality, Archeology and Cultural Heritage (VAST 2007). Brighton, UK.

Massive Software. 2009. Massive. http://www.massivesof tware.com.

Mawson, A. R. 2005. Understanding mass panic and other collective responses to threat and disaster. Psychiatry 68, 2.

McDonald, D., Lazarus, R., Leung, A., And Hussain, T. 2006. Interoperable human behavior models for simulations. In Proceedings of the 2006 Behavior Representation in Modeling and Simulation (BRIMS) Conference. Baltimore, MD.

McKenzie, F. D., Nguyen, Q.-A. H., And Petty, M. D. 2004. Crowd federate architecture and api design. In Proceedings of the Fall 2004 Simulation Interoperability Workshop. 574-587.

Miller, J. H. AND PAGe, S. E. 2007. Complex adaptive systems: an introduction to computational models of social life. Princeton University Press, Princeton and Oxford.

Morini, F., Yersin, B., Maim, J., And Thalmann, D. 2007. Real-time scalable motion planning for crowds. In Proceedings of the International Conference on Cyberworlds (CyberWorlds 2007). Hannover, Germany, 144-151.

Musse, S. R. And Thalmann, D. 2001. Hierarchical model for real time simulation of virtual human crowds. IEEE Transactions on Visualization and Computer Graphics 7, 2, 152-164.

Nguyen, Q.-A. H., McKenzie, F. D., And Petty, M. D. 2005. Crowd behavior cognitive model architecture design. In Proceedings of the 2005 Conference on Behavior Representation in Modeling and Simulation (BRIMS). 55-64.

Ortony, A., Clore, G., And Collins, A. 1988. The Cognitive Structure of Emotion. Cambridge University Press, Cambridge.

Pan, X., Han, C. S., AND LaW, K. H. 2005. A multi-agent based simulation framework for the study of human and social behavior in egress analysis. In Proceedings of the International Conference on Computing in Civil Engineering. Cancun, Mexico, 12-15.

Paris, S., Donikian, S., And Bonvalet, N. 2006. Environmental abstraction and path planning techniques for realistic crowd simulation. Computer Animation and Virtual Worlds 17, 3-4, $325-335$.

Pelechano, N., Allbeck, J., And Badler, N. 2007. Controlling individual agents in highdensity crowd simulation. In Proceedings of ACM SIGGRAPH / Eurographics Symposium on Computer Animation (SCA) 200\%. San Diego, CA, 99-108.

Pelechano, N., Allbeck, J. M., And Badler, N. I. 2008. Virtual crowds: methods, simulation, and control. Morgan \& Claypool.

ACM Journal Name, Vol. V, No. N, Month 20YY. 
Pelechano, N. And Badler, N. 2006. Modeling crowd and trained leader behavior during building evacuation. IEEE Computer Graphics and Applications 26, 6.

Pelechano, N., OBrien, K., Silverman, B., and Badler, N. 2005. Crowd simulation incorporating agent psychological models, roles and communication. In Proceedings of the 1 st International Workshop on Crowd Simulation (V-CROWDS '05). Lausanne, Switzerland.

Pettre, J., de Heras Ciechomski, P., Maim, J., Yersin, B., Laumond, J.-P., and Thalmann, D. 2006. Real-time navigating crowds: scalable simulation and rendering. Computer Animation and Virtual Worlds 17, 445-455.

Pettre, J., Grillon, H., And Thalmann, D. 2007. Crowds of moving objects: Navigation planning and simulation. In Proceedings of the 2007 IEEE International Conference on Robotics and Automation. Rome, Italy.

Petty, M. D., McKenzie, F. D., Gaskins, R. C., And Weisel, E. W. 2004. Developing a crowd federate for military simulation. In Proceedings of the Spring 2004 Simulation Interoperability Workshop (SIW). Arlington, VA, 483-493.

Petty, M. D., McKenzie, F. D., And III, R. C. G. 2003. Requirements, psychological models, and design issues in crowd modeling for military simulation. In Proceedings of the Huntsville Simulation Conference. Huntsville, Alabama.

Presagis. 2009. Ai.implant. http://www.presagis.com/products/simulation/aiimplant/.

Reynolds, C. W. 1987. Flocks, herds and schools: a distributed behavioral model. In Proceedings of the ACM SIGGRAPH '87 Conference. Anaheim, California, 25-34.

SAlzarulo, L. 2006. A continuous opinion dynamics model based on the principle of metacontrast. Journal of Artificial Societies and Social Simulation 9, 1.

Santos, G. And Aguirre, B. E. 2004. A critical review of emergency evacuation simulation models. In Proceedings of NIST Workshop on Building Occupant Movement during Fire Emergencies. University of Delaware, 25-50.

Seyfried, A., Steffen, B., And Klingsch, W. 2005. The fundamental diagram of pedestrian movement revisisted. Jounal of Statistical Mechanics: Theory and Experiment.

Shao, W. And Terzopoulos, D. 2005a. Autonomous pedestrians. In Proceedings of the Eurographics/ACM SIGGRAPH Symposium on Computer Animation (2005). Los Angeles, CA, $19-28$.

Shao, W. And Terzopoulos, D. 2005b. Environmental modeling for autonomous virtual pedestrians. In Proceedings of the 2005 SAE Symposium on Digital Human Modeling for Design and Engineering. 420-433.

Shao, W. And Terzopoulos, D. 2006. Populating reconstructed archaeological sites with autonomous virtual humans. In Proceedings of the 6th International Conference on Intelligent Virtual Agents (IVA 06). Marina del Rey, CA, 420-433.

Silverman, B. G., Bharathy, G., O’Brien, K., and Cornwell, J. 2006. Human behavior models for agents in simulators and games: Part ii: Gamebot engineering with pmfserv. Presence 15, 2, 163-185.

Silverman, B. G., Johns, M., Cornwell, J., and O'Brien, K. 2006. Human behavior models for agents in simulators and games: Part i: Enabling science with pmfserv. Presence 15, 2, 139-162.

Singh, S., Kapadia, M., Faloutsos, P., And Reinman, G. 2009. Steerbench: a benchmark suite for evaluating steering behaviors. Computer Animation and Virtual Worlds 20, 5-6, 533-548.

SIPSER, M. 2005. Introduction to the theory of computation (second edition). PWS Publishing Company, Boston, Massachusetts.

Stauffer, D. 2005. Sociophysics simulations ii: Opinion dynamics. http://www.citebase.org/ abstract?id=oai : arXiv .org: physics/0503115.

Sung, M., Gleicher, M., And Chenney, S. 2004. Scalable behaviors for crowd simulation. Computer Graphics Forum 23, 3, 519-528.

Ting, S. And Zhou, S. 2008. Snap: a time critical decision-making framework for mout simulations. Computer Animation and Virtual Worlds 19, 3-4 (August), 505-514. 
Treullle, A., Cooper, S., And Popovic, Z. 2006. Continuum crowds. In Proceedings of the 33rd International Conference on Computer Graphics and Interactive Techniques (SIGGRAPH'06). Boston, MA, USA, 1160-1168.

Tu, X. and Terzopoulos, D. 1994. Artificial fishes: physics, locomotion, perception, behavior. In Proceedings of the 21st International Conference on Computer Graphics and Interactive Techniques (SIGGRAPH'94). Orlando, FL, USA, 43-50.

Turner, J. C., Hogg, M. A., OAkes, P. J., Reicher, S. D., And Wetherell, M. S. 1989. Rediscovering the social group: A self-categorization theory. The American Journal of Sociology 94, $1514-1516$.

Turner, R. H. And Killian, L. M. 1987. Collective behavior. Prentice Hall, Englewood Cliffs.

Ulicny, B. and Thalmann, D. 2001. Crowd simulation for interactive virtual environments and vr training systems. In Proceedings of the 2001 Eurographics Workshop on Animation and Simulation. 163-170

Yersin, B., Maim, J., de Heras Ciechomski, P., Schertenleib, S., and Thalmann, D. 2005. Steering a virtual crowd based on a semantically augmented navigation graph. In Proceedings of the 1st International Workshop on Crowd Simulation (V-CROWDS'05). 169-178.

Yersin, B., Pettre, J., And Thalmann, D. 2009. Crowd patches: populating large-scale virtual environments for real-time applications. In Proceedings of the 2009 ACM/SIGRAPH Symposium on Interactive 3D graphics and games (I3D 2009). Boston, MA, 207-214.

Received ???; revised ???; accepted ??? 